Meta

Journal des traducteurs

Translators' Journal

\title{
Quelques aspects de la datation de termes techniques : le cas de l'enregistrement et de la reproduction sonores
}

\section{John Humbley}

Volume 39, numéro 4, décembre 1994

Hommage à Bernard Quemada : termes et textes

URI : https://id.erudit.org/iderudit/003157ar

DOI : https://doi.org/10.7202/003157ar

Aller au sommaire du numéro

Éditeur(s)

Les Presses de l'Université de Montréal

ISSN

0026-0452 (imprimé)

1492-1421 (numérique)

Découvrir la revue

Citer cet article

Humbley, J. (1994). Quelques aspects de la datation de termes techniques : le cas de l'enregistrement et de la reproduction sonores. Meta, 39(4), 701-715. https://doi.org/10.7202/003157ar
Résumé de l'article

La datation de termes techniques se fait souvent de la même manière que celle des mots de la langue générale : par dépouillement d'un corpus littéraire ou peu spécialisé. Une méthode complémentaire consiste à identifier la technique en question et à suivre son évolution en s'attachant d'abord à la réalité, puis aux termes qui la dénomment. L'invention du phonographe offre une illustration de cette démarche : on identifie les étapes de sa découverte, on dépouille les documents techniques et de vulgarisation qui y font référence, et on arrive à des datations qui ne sont pas seulement antérieures à celles des dictionnaires, mais qui ont aussi une cohérence interne. L'approche terminologique peut donc fonctionner aussi en diachronie.
Tous droits réservés @ Les Presses de l'Université de Montréal, 1994
Ce document est protégé par la loi sur le droit d'auteur. L’utilisation des services d'Érudit (y compris la reproduction) est assujettie à sa politique d'utilisation que vous pouvez consulter en ligne.

https://apropos.erudit.org/fr/usagers/politique-dutilisation/ 


\title{
QUELQUES ASPECTS DE LA DATATION DE TERMES TECHNIQUES : LE CAS DE L'ENREGISTREMENT ET DE LA REPRODUCTION SONORES
}

\author{
JOHN HUMBLEY
}

Université Paris-Nord, Villetaneuse, France

\begin{abstract}
Résumé
La datation de termes techniques se fait souvent de la même manière que celle des mots de la langue générale : par dépouillement d'un corpus littéraire ou peu spécialisé.

Une méthode complémentaire consiste à identifier la technique en question et à suivre son évolution en s'attachant d'abord à la réalité, puis aux termes qui la dénomment. L'invention du phonographe offre une illustration de cette démarche : on identifie les étapes de sa découverte, on dépouille les documents techniques et de vulgarisation qui y font référence, et on arrive à des datations qui ne sont pas seulement antérieures à celles des dictionnaires, mais qui ont aussi une cohérence interne.

L'approche terminologique peut donc fonctionner aussi en diachronie.
\end{abstract}

\begin{abstract}
Dating technical terms is often carried out in the same way as for words of the general language: by finding references in literary or non-specialized works.

One complementary method would be to identify the technique in question, and to trace how it evolved terminologically: by first examining the historic reality and then the terms which named it. The invention of the phonograph provides an illustration of this sort of dating, by identifying the stages of its discovery, by going through relevant technical and popular scientific publications of the time, to make it possible not only to antedate dictionaries, but also to obtain an internal consistency in the dating.

The terminological approach can thus also work in diachrony.
\end{abstract}

\section{TERMINOLOGIE DIACHRONIQUE ET DATATIONS}

En terminologie et en terminographie, la démarche classique est avant tout conceptuelle, telle qu'elle est décrite et illustrée par Eugen Wüster. Même là où elle est contestée, cette méthode constitue un point de référence auquel toute autre approche se compare. Or, Wüster concevait sa méthode dans le cadre de la normalisation technique internationale, et même si les aspects linguistiques du travail étaient loin d'être négligés, sa visée était moins la langue que les concepts qu'il s'agissait de nommer et de définir. Il peut paraitre curieux dans ce cas de vouloir appliquer sa méthode aux datations, recherche dont l'orientation peut paraître à la fois linguistique et historique. Mais comme Bernard Quemada n'a cesse de dire dans ses séminaires, l'étymologie fait partie non pas des aspects linguistiques, mais des connaissances encyclopédiques présentées dans un dictionnaire. Partant de ce principe, il peut être utile de concevoir les datations du vocabulaire scientifique et technique comme un exercice de terminologie diachronique, ou de néologie rétrospective, pour reprendre le terme de Gabrielle Quemada. Prenant comme point de départ les concepts, comme le faisait Wüster, on examine les différentes façons de les nommer. En suivant cette méthode, nous faisons en même temps une relecture de Louis 
Guilbert, à la lumière de la terminologie de l'époque de Wüster, sans oublier les pratiques actuelles.

Le vocabulaire de l'enregistrement et de la reproduction sonores constitue un terrain d'enquête favorable pour illustrer la démarche suggérée. D'une part, il s'agit d'une innovation incontestable: la reproduction du son a marqué un tournant que l'on peut qualifier d'historique, car depuis la réalisation d'Edison on dispose non seulement de documents écrits, mais aussi d'archives sonores. D'autre part, l'histoire a voulu que la description de cette invention soit bien documentée à la fois en français et en anglais, car, si c'est un Américain qui l'a réalisée, c'est un Français qui l'avait conçue. Nommer l'invention et ses parties relève donc en partie d'une histoire d'échange entre ces deux communautés linguistiques. Toute invention, cependant, est l'héritière de techniques antérieures: on innove à partir de l'ancien, et les termes employés reflètent cet ancrage dans le passé. L'invention du phonographe n'échappe pas à cette règle.

Une motivation supplémentaire de cette étude a été fournie par un article récent (Wooldridge 1990-1991), faisant part d'une série de datations recueillies dans une revue des arts et des techniques des années 1920-1930. L'auteur fait remarquer que la lecture d'une revue un peu spécialisée permet de reculer de façon significative les datations retenues dans les grands dictionnaires français, et le hasard a voulu que plusieurs numéros de cette revue portent sur l'enregistrement sonore. Si un dépouillement aléatoire permet en effet d'apporter des éléments nouveaux, il ne faudrait pas s'arrêter en si bon chemin, car on peut espérer qu'une recherche orientée vers l'évolution d'une nouveauté technique dans la documentation appropriée apporte encore davantage d'informations. Au lieu de privilégier les mots, on adopte l'approche du terminologue, et en même temps, de l'historien, et on tente de reconstruire l'historique de l'invention, et de bien cerner les concepts en jeu. Bien sûr, l'aspect linguistique n'est pas perdu de vue, et on étudie systématiquement les dénominations des inventions ainsi que les adaptations éventuelles réalisées lors la vulgarisation. Comme en terminologie donc, on part du concept, et on étudie par la suite comment il est exprimé, par qui et dans quel contexte. Une enquête plus approfondie, qui dépasserait celle des simples datations, tiendrait également compte de la combinatoire et de l'expression des fonctions, qui trouvent néanmoins un écho dans les lignes qui suivent.

\section{L'HISTOIRE DE L'INVENTION}

Nous plaçons l'essentiel de notre étude dans le bref laps de temps d'à peine une année, de 1877 à 1878 , qui a vu l'invention effective du phonographe et de sa présentation au public en France ainsi qu'à l'étranger. Il n'est pas inutile de rappeler brièvement l'histoire de ce qui ressemble fort à une double invention. On sait que Thomas Edison, qui s'était donné pour but d'enregistrer le morse, avait réalisé dans ses «laboratoires» de New Jersey en 1877 un instrument qui permettait d'enregister et de reproduire la voix humaine, invention qu'il a tout de suite brevetée. $\grave{A}$ peine quelques mois auparavant, le poète français Charles Cros avait déposé à l'Académie des Sciences une enveloppe cachetée qui contenait la description d'un appareil qui ressemblait fortement à celui d'Edison, mais qui, faute de moyens, n'avait pu être construit. Il s'agissait, selon le titre de son article, daté du 18 avril 1877, d'un «procédé d'enregistrement et de reproduction». La paternité de l'invention fut fort controversée à l'époque, et la question n'est pas complètement réglée aujourd'hui, d'autant plus que l'auteur d'une découverte antérieure, Émile Scott de Martinville, se mettait sur les rangs pour être reconnu à son tour comme véritable père de cette innovation. Contrairement aux instruments d'Edison et de Cros, cependant, l'appareil de Scott de Martinville, réalisé en 1857 , ne cherchait pas à reproduire le son, mais à transcrire les vibrations sonores de la voix de telle façon que l'on puisse les interpréter en 
les regardant. La parenté entre les trois inventions est néanmoins manifeste, ce qui illustre, s'il en était besoin, la relativité de la nouveauté.

Quoi qu'il en soit, le phonographe d'Edison, après avoir fait sensation partout où il a été montré, et notamment à l'Exposition universelle de Paris de 1878, a rapidement disparu du regard public et n'a fait sa réapparition que dix ans plus tard, sous trois formes différentes, perfectionné par l'inventeur du téléphone, Alexander Graham Bell, par Thomas Edison lui-même, et par Émile Berliner, Allemand émigré aux États-Unis, inventeur du gramophone, ou phonographe à disque. Certains documents de cette deuxième période ont été retenus également pour témoigner de l'implantation réelle des néologismes de $1877-1878$.

\section{LES SOURCES : LA DOCUMENTATION}

Le non-spécialiste du domaine commence ses recherches par les ouvrages secondaires (voir 7.3), principalement de l'histoire de l'invention. Non seulement il obtient ainsi une vue d'ensemble épurée par le recul chronologique, mais il peut aussi accéder aux sources primaires. Certains ouvrages modernes sont particulièrement précieux du point de vue de la documentation, dont La Machine parlante de Paul Charbon, qui comporte des reproductions en fac-similé des textes manuscrits de Charles Cros, et nous nous sommes appuyé sur l'ensemble des bibliographies lors de la recherche des documents d'époque, qui, seuls, font l'objet d'un dépouillement (7.2).

Si l'on entend par sources primaires les écrits des inventeurs eux-mêmes, on doit constater que, pour cette époque héroïque, elles sont très rares et parfois quelque peu suspectes. Nous savons, par exemple, que le fameux croquis du prototype du phonographe, indubitablement de la main d'Edison, qui porte l'inscription laconique «Kreusi, make this!», serait un faux : l'inventeur aurait ajouté cette mention bien plus tard. En revanche, les sources de première divulgation sont généralement très accessibles, car conservées en tant que telles, ou encore reproduites dans d'autres publications de l'époque. Ce type de source constitue l'essentiel du corpus de cette recherche. On se situe donc, comme souvent en terminologie, au niveau de l'échange entre spécialistes de domaines connexes, les revues concernées étant La Nature ou, plus tard, La Lumière électrique. En revanche, on tient compte aussi des descriptions de l'invention qui relèvent davantage de la vulgarisation dans la presse d'intérêt plus général ou du moins non spẹcialiste, comme le Magasin pittoresque, L'Illustration, Le Figaro, voire dans La Semaine du Clergé, où l'invention de Charles Cros fut révélée au public pour la première fois, par un nommé Lenoir, pseudonyme pour Le Blanc. Les premières monographies consacrées à cette invention, enfin, paraissent au cours de cette période, ce qui permet de compléter rapidement une première documentation. Un premier dépouillement ne révèle pas une grande différence entre le vocabulaire des inventeurs ou de leurs premiers divulgateurs et celui de la vulgarisation: en effet, les seconds se limitent aux aspects les moins techniques, et se concentrent sur l'innovation elle-même.

\section{STRUCTURATION PROVISOIRE DU CHAMP CONCEPTUEL}

L'approche terminologique demande une visualisation de la structuration de la matière traitée, réalisée généralement sous forme de l'arbre du domaine ou d'un schéma équivalent. On peut l'exprimer ici en termes de fonctions remplies par différentes parties de l'instrument.

0. L'invention

1. Enregistrer

1.1 capter le son

1.2 transmettre le son 
1.3 inscrire le son

1.3.1 par un instrument

1.3.2 sur un support

1.3.3 comportant un récipient

1.4 mettre en mouvement le support

1.4.1 par une source d'énergie

1.4.2 transmise au support

2. Reproduire le son

2.1 capter le son enregistré

2.2 transmettre le son enregistré

2.2.1 par le corps de résonance

2.3 mettre en mouvement le support

2.4 augmenter le volume

\section{Exploitation}

Nous complétons ci-dessous ce tableau avec les mots ou les expressions recueillis, reproduits en dernière partie avec les datations correspondantes, où l'ensemble des attestations est présenté.

\section{0 . Invention}

phonographe

machine parlante

appareil

appareil enregistreur

appareil reproducteur

\section{1. enregistrer}

enregistrer

graver

1.1 capter le son

embouchure

conque, cuve

embouchure du porte-voix

virole

\section{2 transmettre le son}

tympan

membrane métallique

membrane analogique à celle du téléphone

1.3 inscrire le son

ressort porte-style

style

pointe métallique

pointe

pointe traçante

lame métallique

\subsection{1 par un instrument porte-voix}

\subsection{2 sur un support}

cylindre

cliché phonographique

cliché

lame parlante

phonographie

enregistrement 


\subsection{3 qui comporte un récipient rainure hélicoïdale cannelure en forme d'hélice sillon hélicoïdal sillon}

1.4 mettre en mouvement le support tourner manivelle ressort pédale

2. reproduire le son reproduire reproduction

2.1 capter le son enregistré style aiguille

\section{2 transmettre le son enregistré}

2.2.1 par le corps de résonance membrane téléphone

2.3 mettre en mouvement le support (tourner la) manivelle

2.4 augmenter le volume cornet

\section{Exploitation}

collection

casier

album

dictionnaire parlant

\section{FORMATION DU VOCABULAIRE DE LA REPRODUCTION SONORE}

Les résultats de ce premier dépouillement posent quelques questions pratiques, dont le triple découpage bien connu des terminologues. Il s'agit d'abord de découper la chaîne syntagmatique pour connaître les limites du terme, ensuite de le découper dans le discours pour préciser le passage à la langue, enfin de découper dans la spécialité pour savoir quels mots appartiennent au domaine concerné. Cette phase délicate de terminologisation et de lexicalisation est rendue moins difficile à saisir par le recul qu'a le lecteur de la fin du vingtième siècle, qui sait, lui, quels mots sont effectivement devenus des termes, et quels mots ont été vulgarisés. Il s'agit cependant de pistes et non pas de preuves, cependant, car il est également possible qu'un mot soit terminologisé pour un moment seulement, et seule la recherche de documentation historique peut résoudre ce problème.

\subsection{Morphologie}

Les procédés de composition et de dérivation figurent assez peu dans notre vocabulaire technique proprement dit; on en relève des exemples importants en revanche dans le vocabulaire vulgarisé, et en premier lieu dans le cas de la dénomination de l'invention elle-même, où la composition savante est consciemment privilégiée.

\subsubsection{Composition savante}

On sait que la composition savante phonographe s'est vite imposée pour désigner l'invention. C'est le mot qu'avait choisi Edison, et le succès de sa réalisation a certainement 
assuré la vulgarisation du mot, tant sur le continent nord-américain qu'en Europe. Comme c'est souvent le cas des éléments gréco-latins, il est difficile de savoir dans quelle langue elle fut réalisée pour la première fois, si les mots anglais et français ont la même origine ou s'il s'agit, comme pour l'invention elle-même, de deux créations parallèles. En effet, le mot phonographe avait été utilisé en français avant la réalisation d'Edison et avant l'invention virtuelle de Cros, comme le fait remarquer son premier divulgateur:

«M. Charles Cros, l'inventeur véritable de l'instrument que nous avions appelé le phonographe, mais qu'il appelle le paléophone (voix ou parole du passé), attendu que le mot phonographe, très bon assurément, avait été déjà employé pour autre chose.» Le Blanc, janvier 1878 , p. 440

Nous savons que paléophone, autre composition savante, que Cros n'emploie pas dans sa première description, n'a jamais été vulgarisé, contrairement à phonographe, que l'on relève dans la presse quotidienne et hebdomadaire tout au long de l'année 1878.

Comme le suggère Le Blanc, c'est à l'inventeur de nommer son invention; cette vision performative des choses trouve des échos ailleurs, y compris dans les textes de vulgarisation:

«C'est à l'Américain M. Edison, de Menlo-Park (New-Jersey), que l'on doit le nouvel appareil, auquel il a donné le nom de Phonographe.» Magasin pittoresque, 46, octobre 1878, p. 343.

Ce type de composition fut également employé par Scott de Martinville, qui avait appelé son invention le phonautographe, que l'on peut dater de 1857. Cet inventeur ne négligea pas non plus les possibilités de dérivation de la composition savante, car il avait intitulé sa présentation de 1857 Principes de phonautographie. De même, on relève dans la monographie de Niaudet (1878 mais voir Références) le dérivé phonographie. Scott de Martinville, en désaccord sur le principe même de l'invention de Cros et d'Edison, qu'il mettait dans le même sac, s'interroge sur le choix de la dénomination de l'enregistrement, phonautographie ou phonographie.

L'adjectif phonographique est également attesté à cette époque.

Les possibilités de dérivation furent encore plus amplement explorées dix ans plus tard dans La Nature, comme on le voit sous phonographier, parmi les datations (6).

Suivre le fil des dérivations peut cependant induire en erreur: les mêmes mots ne désignent pas nécessairement la même chose. Le «phonautographe» de Berliner n'a rien à voir avec l'invention de Scott de Martinville, car il s'agissait de l'appareil enregistreur, et phonographie, ici un dénombrable, est un disque, et non pas un principe.

Phonogramme, désignation générale de tous les supports sonores, semble dater de cette seconde époque, lorsque le disque commence à concurrencer le cylindre. La première citation que nous relevons fait référence au disque, la seconde au cylindre.

\subsubsection{Autres cas de composition}

La composition savante n'était pas la seule façon de dénommer l'invention, et les vulgarisateurs ont également recours à des composés dont certains se sont lexicalisés un certain temps:

machine parlante: il s'agit peut-être d'une traduction de l'anglais talking machine, dénomination courante lors des démonstrations d'Edison. Elle est encore plus courante dix ans plus tard, lorsque le besoin d'un terme englobant fut ressenti à cause de l'apparition de différents types de perfectionnement, portant des noms différents (phonographe, graphophone, gramophone). On relève à l'occasion des formes redondantes, telles que phonographe parlant. 
Certaines désignations peuvent avoir une visée polémique, comme appareil répétiteur sous la plume de Scott de Martinville, qui contestait la légitimité de la démarche, peu scientifique à ses yeux.

La composition est par ailleurs peu exploitée, ressort porte-style est relevé mais ne semble pas avoir eu de suite:

«Deux étouffoirs X, formés par des morceaux de tube de caoutchouc, amortissent les vibrations de la membrane et celle du ressort porte-style.» Niaudet, 1887 p. 157-158.

Ici, au moins, l'emploi de la construction $\mathrm{N}+\mathrm{N}$ ne doit rien à l'anglais.

\subsection{Nouveaux emplois de mots existants}

En quoi consiste alors la néologie de ce vocabulaire? Puisque dans la réalité il s'agissait de faire du neuf avec de l'ancien (l'impression de bricolage que donne la citation précédente n'est pas exceptionnelle), on employait les mots d'autres domaines dans ce qui allait devenir un secteur d'activités important. La plupart des termes en devenir sont tout simplement ce que Tournier $(1985: 210)$ appelle des glissements de domaine d'application ou d'environnement, ce que Guilbert avait dénommé plus simplement les nouveaux emplois. Ils sont nombreux dans notre corpus :

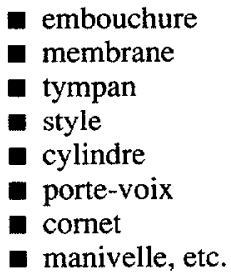

L'emploi de cylindre comme néologisme de domaine d'emploi illustre le nombre relativement élevé d'opérations sémantiques impliquées dans ce processus. Le point de départ représente déjà une étape dans cette évolution: d'abord employé comme figure de géométrie, y compris dans ses premières attestations en français, cylindre sert à désigner des objets concrets ayant cette forme. Il était donc disponible pour désigner le support de l'enregistrement des premiers appareils, format qui persiste d'ailleurs en France jusqu'à l'époque de la Première Guerre mondiale et encore plus tard sur le continent américain. D'autres candidats auraient été possibles, comme rouleau, effectivement employé par la suite, mais cylindre est la seule dénomination effectivement relevée dans ce corpus provisoire. Dans les premiers textes il dénomme à la fois le mandrin, sur lequel on posait le support de l'enregistrement, et ce support même.

\subsection{Métaphores}

Trois métaphores dominent le processus de néologie de cette nouvelle technique, prises dans trois techniques antérieures qui présentent des analogies soit de fonction, soit de moyen. La plus ancienne est celle de l'écriture, dont la prégnance reste si forte dans le domaine sonore qu'elle détermine non seulement les mots, mais aussi la façon de voir les choses. En effet, à force de filer la métaphore de l'écriture, les inventeurs finissent par se méfier de tout ce qui en départ. C'est ainsi que Scott de Martinville proteste que le phonographe d'Edison, comme celui de Charles Cros, n'est qu'une machine à répéter, et que celle que lui avait conçue était une machine à écrire le son.

L'analogie est tout à fait consciente sous la plume de plusieurs auteurs, comme on peut en juger des extraits suivants, où les mots désignant l'écriture sont marqués en gras. 
«La majorité du public lisant ne sait pas qu'il existe un appareil français, non pour répéter (1), mais encore pour écrire la parole, selon la signification du mot phonographe.» Scott, 1878, p. 4 .

«un style, chargé d'écrire, placé convenablement pour toucher un peu obliquement le plan de la couche sensible» Scott, 1857, cité en 1878, p. 17

«On doit à M. Scott de Martinville l'idée d'employer les membranes comme intermédiaires pour l'inscription des sons transmis par l'air. Niaudet, 1878, p. 150.

«pendant l'inscription et pendant la reproduction du son», Niaudet, 1878, p. 160.

L'emploi de enregistrer et enregistrement relève également de la métaphore de l'écriture. Charles Cros l'employa dans le titre de son papier:

«procédé d'enregistrement et de reproduction des sons» (18 avril 1877)

Niaudet l'emploie également pour les tentatives antérieures de reproduction sonore:

«Thomas Young décrit dans un ouvrage daté de 1807 (1) un instrument composé d'un cylindre animé à la fois d'un mouvement de rotation et de translation et destiné à l'enregistrement des vibrations sonores.» Niaudet, 1878, p. 148

«Un instrument capable d'enregistrer les sons» Niaudet, 1878, p. 156

«On peut voir que M. Leblanc [Semaine du clergé] décrit à cette date et sous le nom même de Phonographe un appareil identique à celui de M. Edison, sauf que l'enregistrement ne s'y fait pas sur papier d'étain.» Scott, 1878, p. 4

«Le phonographe d'Edison, merveilleux au point de vue scientifique, ne paraît pas susceptible de beaucoup d'applications dans son état actuel au point de vue pratique. Il faut, pour que le son s'enregistre, parler dans une embouchure... Moniteur universel, 6 mai 1878, p. 729.

L'instrument de l'enregistrement, généralement dénommé stylet ou style, qui rappelle soit l'écriture, soit encore la gravure.

La deuxième série de métaphores concerne précisément la gravure, inaugurée par la toute première présentation de «Le Blanc»:

«Supposons enfin que l'on traite la surface métallique, après qu'elle a reçu les impressions vibratiles du petit ressort, par un procédé délicat analogue à celui au moyen duquel les aquafortistes exécutent leurs gravures à l'eau forte.» Le Blanc, 1877, p. 1625

Cette technique n'était pas celle qu'Edison utilisait, comme le fait remarquer un chroniqueur de 1888 :

«Le gauffrage doit être abandonné pour un procédé de gravure, c'est-à-dire qu'au lieu de refouler la surface de l'enregistrement avec un style comme dans le phonographe original, il faut produire une tracé parallèle à la surface d'inscription» «La phonogravure et le gramophone». La Nature 786, 1888, p. 49.

«On sait qu'Edison enregistrait les vibrations sonores en produisant au moyen d'une pointe mousse, reliée à un diaphragme vibrant, des déformations dans une feuille d'étain qui était pour ainsi dire repoussée; MM. Bell et Tainter, au contraire /.../ emploient un stylet tranchant qui grave ou découpe sur un cylindre, recouvert de cire d'abeilles ou de paraffine, un sillon à peine visible.» J. Wetzler, «États-Unis», La Lumière électrique, 11.10.1888, p. 290.

Les procédés qualifiés ici de «gravure» ne sont pas identiques: le premier auteur fait référence à Émile Berliner, l'inventeur du gramophone, qui mit en pratique un procédé similaire à celui de Charles Cros, surtout en ce qui concerne l'emploi d'acide. Le second décrit le graphophone de Bell, qui ne l'emploie pas. La différence de procédé 
cependant n'empêche pas les vulgarisateurs d'employer cette métaphore, surtout sous la forme du verbe graver:

«L'opérateur gravant par la parole un cliché phonographique», L'Illustration, 1830, 3.03.1878, p. 196.

«L'opérateur faisant répéter par l'appareil les paroles gravées sur le cliché», L'Illustration, $1830,3.03 .1878$, p. 196.

Comme le montre cette dernière attestation, la métaphore la plus utilisée dans la vulgarisation est celle de la photographie, elle-même tributaire de la gravure. Le Blanc, dans sa première présentation de l'invention de Charles Cros, justifiait par cette analogie la composition savante de phonographe, dont la sonorité rappelait photographe. La métaphore du cliché photographique est encore plus souvent relevée, d'abord sous la plume de Le Blanc, mais aussi dans la presse générale.

Malgré les nombreuses attestations de cliché/clicher en 1877-1878, cet emploi verbal n'a pas été lexicalisé dans ce contexte.

\subsection{Métonymie}

Parmi les opérations métasémiques productives en néologie, on met en bonne place la métonymie. Elle n'est pas négligée dans l'histoire du vocabulaire de l'enregistrement, comme le montre l'étude du mot... enregistrement.

Enregistrement est d'abord le résultat d'une métaphore : on «enregistre» un discours oral comme on enregistre de l'écrit. On note l'insistance qu'emploient les premiers divulgateurs lorsqu'ils présentent le phonographe comme instrument qui enregistre et qui reproduit la parole. Si l'accent avait été mis sur la musique, on peut se demander si la métaphore de l'enregistrement aurait été employée.

Quoi qu'il en soit, c'est surtout le verbe enregistrer que l'on relève :

«Un instrument capable d'enregistrer les sons...» Niaudet, 1878, p. 156.

«Il faut, pour que le son s'enregistre, parler dans une embouchure», Moniteur universel, 6 mai 1878 , p. 729.

Niaudet parle de l'appareil enregistreur, (Niaudet, 1878 p. 156), expression relevée dix ans plus tard dans La Nature 1888, p. 50.

Le nom enregistrement est employé d'abord comme l'acte d'enregistrer:

«Ce papier est suspendu au-dessus du vide présenté par la rainure hélicoïdale, et c'est dans cette partie que se fera l'enregistrement, comme nous allons le dire», Niaudet, 1878, p. 157.

La métonymie intervient dès lors que le résultat est désigné par le nom du processus, cas très fréquent de ce genre de métasémie. C'est le cas de enregistrement qui désigne l'objet (phonogramme), dès 1888 .

\subsection{Paradigme désignationnel}

La démarche terminologique ne serait pas complète si l'on n'examinait pas toutes les dénominations utilisées pour le même concept. En effet, si l'une finit généralement par s'imposer, c'est seulement après un certain foisonnement néologique, comme nous l'avons vu pour enregistrer et phonographe. Il existe plusieurs types de synonymes néologiques. Le premier est celui des synonymes stricts de la même réalité : ainsi ce que Le Blanc avait baptisé phonographe, Cros lui-même l'appelait paléographe. Le deuxième type de synonymie représente les dénominations des variantes de l'invention, qui foisonnent dans la deuxième période (phonographe, graphophone, gramophone). Le troisième cas 
est celui des hyperonymes: machine parlante, et des anaphores lexicalisables : appareil, qui devient à partir de 1889 un générique, représentant appareil enregistreur et appareil reproducteur, réunis ou non dans le même instrument.

C'est le cas également de sillon. On relève comme termes concurrents rainure, rainure hélicoïdale, spirale... Sillon s'est imposé et se trouve dès les premiers documents sous d'autres formes, tel le verbe sillonner.

«Les vibrations de cette pointe, par une ingénieuse combinaison, viennent s'imprimer sur une feuille de tain [sic] enveloppant un cylindre dont l'axe est un écrou qui correspond aux spirales sillonant le cylindre.» Le Figaro, 14 mars 1878, p. 2.

\section{CONCLUSION}

La méthode de datation proposée ici ne constitue en aucune manière une critique voilée des dictionnaires en général et du Trésor de la langue française en particulier. Il s'agit tout simplement de suggérer des outils plus appropriés à l'analyse de la langue de spécialité dans sa dimension historique. Si l'on souhaitait mener à bien une telle recherche sur l'ensemble des spécialités que contient un dictionnaire, il serait nécessaire, comme en terminologie contemporaine, de confier une part importante de ces travaux aux spécialistes eux-mêmes, en l'occurrence, les historiens des sciences et techniques.

\section{DATATIONS}

Sont présentés ici les termes principaux du vocabulaire de la phonographie, tels qu'ils ont été dégagés au cours du dépouillement décrit ci-dessus. Les néologismes marginaux, qui ne représentent qu'une occurrence dans un nouveau domaine d'emploi (mandrin, manivelle, etc), ne sont pas repris ici.

Les termes qui n'ont pas été lexicalisés sont reproduits entre parenthèses.

\section{album}

«... vous pouvez avoir dans votre salon un phonographe avec un casier contenant un album de matières phonographiques triées sur le volet. Vous pouvez prendre un feuillet dans l'album, le placer dans le phonographe et écouter une symphonie.»

Le Figaro, 12 avril 1878, p. 1

La datation de 1928 fournie par Wooldridge (1990-1991 p. 96) est plus proche de la réalisation effective des albums de disques. En 1878 il s'agissait encore d'une vue de l'esprit: il conviendrait de noter qu'il s'agit ici d'un hapax, et que la véritable datation serait à chercher plutôt vers 1905 , lorsque les premiers albums furent effectivement commercialisés.

appareil

«L'opérateur faisant répéter par l'appareil les paroles gravées sur le cliché» (illustration). L'Illustration, 3 mars 1878 , p. 196

Le passage du générique appareil d'un anaphorique à une dénomination pose des problèmes de principe: il fait partie du paradigme dénominatif, et ne serait pas porté comme datation.

(appareil enregistreur)

Niaudet, 1878, p. 156; La Nature, 786, 1888, p. 50

(appareil reproducteur)

La Nature, 786, 1888, p. 49

(appareil parleur)

La Nature, 786,1888 , p. 49 
casier

voir album

cornet

«L'Académie a entendu sortir du fond du cornet la reproduction de toutes les paroles sorties de la bouche de l'opérateur [...]»

L'Illustration, 1830, 3 mars 1878, p. 196

Le TLF donne une attestation de cornet d' un phonographe dès 1883.

(cliché)

«Que résulte-t-il de tout cela? Il en résultera qu'on obtiendra un cliché, soit un cylindre»,

Le Blanc, La semaine du clergé, X, 51, 10 octobre 1877, p. 1625

«Un cliché /.../ se conservant pour toujours comme se conservent les clichés sur bois ou sur cuivre».

Le Blanc, La semaine du clergé, X, 51, 10 octobre 1877, p. 1625

«Un phonographe avec clichés variés : un air de la Patti...»

titre d'un dessin humoristique, L'Illustration, 1833, 13.04.1878, p. 245. Le soprano, Adelina Patti, célébrée par Alfred de Musset, n'enregistrera pas avant 1905 !

(clicher)

«Devant la Société de physique de Londres avec un phonographe à deux embouchures, on a pu clicher et ensuite reproduire un duo.»

W. de Fonville, L'Illustration, 1831, 30 mars 1878 , p. 210

«Grève de MM. les orateurs: On s'en fiche pas mal à la Chambre, maintenant qu'on a le phonographe. On leur servira de vieux discours clichés par l'instrument.»

L'Illustration, $1833,13.04 .1878$, p. 245

cylindre

1) le mandrin

«La surface du cylindre présente une rainure hélicoïdale... On y colle une feuille mince d'étain.»

Niaudet, 1878 , p. 157

2) le support de l'enregistrement

«un cliché, soit un cylindre»

Le Blanc, 1877 , p. 1625

«Le cylindre phonographique ou phonogramme...»

Richard, 1889 , p. 360

Dans les premiers textes, cylindre peut désigner soit le support même de l'enregistrement, la feuille d'étain pour le phonographe d'Edison, soit le mandrin qui le soutient. Dans certaines descriptions, d'ailleurs, on passe imperceptiblement du second au premier. La datation de 1905 du $T L F$ peut donc être reculée à 1877.

(dictionnaire parlant)

«Ces lames [supports d'enregistrement], qui peuvent être fabriquées à très bon marché, rendront de réels services pour l'étude des langues étrangères, car elles permettent de conserver indéfiniment et de se faire dire à loisir les mots dont la prononciation trop difficile nécessite des répétitions. [...] On arrivera à constituer aussi un véritable dictionnaire parlant, problème dont la solution ne pouvait être espérée ni même prévue il y a quelques années.»

E. Hospitalier, «Les lames parlantes de M. Lambrigot», La Nature, 309, 3 mai 1879, p. 350 disque

«Le dispositif auquel M. Berliner s'est arrêté, comprend un moteur pouvant faire tourner un disque circulaire en verre d'une épaisseur d'environ 5 millimètres et d'un diamètre de 275 millimètres.»

J. Wetzler, La Lumière électrique, 10 décembre 1887, p. 542 
Selon l'approche conceptuelle, il convient de retenir cette attestation comme celle qui introduit en français ce nouvel emploi de disque, car c'est l'invention de Berliner qui sera généralisée. Charles Cros avait déjà envisagé la possibilité d'enregistrer sur un disque, mais il parle de «spirale plane» dans sa description du 16 avril 1877. L'année 1887 est donc raisonnable comme conjonction entre la dénomination et l'objet qui devra se généraliser.

Le $T L F$ date ce sens de disque en français de 1918, mais disk/disc en anglais dès 1888, et s'interroge sur la possibilité d'emprunt. Même si dans l'attestation ci-dessus la date est antérieure, il s'agit de la description d'une invention réalisée aux États-Unis.

\section{enregistrement}

1) le fait d'enregistrer

«procédé d'enregistrement et de reproduction des phénomènes perçus par l'ouïe»

manuscrit de Charles Cros, 16 avril 1877, dans Charbon, 1981, p. 23

«Thomas Young décrit dans un ouvrage daté de 1807 (1) un instrument composé d'un cylindre animé à la fois d'un mouvement de rotation et de translation et destiné à l'enregistrement des vibrations sonores.»

Niaudet, 1878 , p. $148-149$

2) le support de l'enregistrement

«Le gauffrage doit êtrè abandonné pour un procédé de gravure, c'est-à-dire qu'au lieu de refouler la surface de l'enregistrement avec un style comme dans le phonographe original, il faut produire un tracé parallèle à la surface d'inscription»

«La phonogravure et le gramophone». La Nature, 786,1888 , p. 49

Le $T L F$ ne date pas ces deux sens: on peut postuler 1877 pour le premier sens et 1888 pour le second.

\section{enregistrer}

«Un instrument capable d'enregistrer les sons».

Niaudet, 1878 , p. 156

«Il faut, pour que le son s'enregistre, parler dans une embouchure»

Moniteur universel, 6 mai 1878 , p. 729

La datation du TLF (XX' $\mathrm{XX}^{\mathrm{e}}$ siècle) peut être ramenée à 1878 .

enregistreur

voir appareil enregistreur

graver

«L'opérateur gravant par la parole un cliché phonographique»,

L'lllustration, 1830, 3.03.1878, p. 196

«L'opérateur faisant répéter par l'appareil les paroles gravées sur le cliché»,

L'Illustration 1830, 3.03.1878, p. 196

Dans les attestations de Wooldridge, graver et gravure semblent être employés comme des métaphores vivantes, ce qui suggère que celles des années 1870 ne furent pas lexicalisées.

(lame parlante)

«M. Lambrigot place ses lames sur une caisse résonnante et les phrases peuvent être perçues par plusieurs personnes à la fois, sans le secours d'un cornet.»

E. Hospitalier, «Les lames parlantes de M. Lambrigot», La Nature, 309, 1879, p. 350 


\section{machine parlante}

«Nous avons indiqué quelques-uns des usages de la nouvelle machine parlante, mais vraiment dans ce pays de la libre parole, il est impossible de prédire jusqu'où iront ses nombreuses applications.»

Le Figaro, 14 mars 1878, p. 2

«Le phonographe d'Edison, merveilleux au point de vue scientifique, ne paraît pas susceptible de beaucoup d'applications dans son état actuel au point de vue pratique. [...] À bientôt la nouvelle machine parlante.»

Le Moniteur universel, 6 mai 1878 , p. 729

Machine parlante ne semble pas avoir fait l'objet d'une entrée dans le $T L F$.

phonogramme

«On mettra en vente un grand nombre d'appareils parleurs identiques de construction, d'une manipulation facile et d'un prix peu élevé. Les acquéreurs de ces appareils pourront se procurer une collection sans cesse croissante de phonogrammes reproduisant des écrits, des chants...»

La Nature, 786, 1888, p. 51

«Le phonogramme reçoit son mouvement d'une pédale...»

La Lumière électrique, 32, 25 mars 1889, p. 358

«Le cylindre phonographique ou phonogramme...»

Richard, 1889, p. 360

La mention «vieilli» du TLF n'est pas exacte: sa valeur de terme générique, établie d'après ces attestations dès 1888 , vaut encore de nos jours, même si elle est peu connue du public. En 1994, l'organisation des fabricants de «disques» s'intitule le Syndicat national des éditeurs de phonogrammes (Snep), et il existe également l'Union des producteurs de phonogrammes français indépendants (Upfi). Les journalistes glosent le plus souvent, $c f$. «...l'industrie du disque a réalisé en France un chiffre d'affaires de 6,1 milliards de francs..., pour un total de 130 millions de «phonogrammes» (disques, cassettes, DCC et mini-disques confondus : 7,47 \% en volume)». Libération, 29/30.01.1994, p. 3, col. 1, Paul Loubière.

phonographe

«Par cet instrument que nous appellerons, si nous étions appelé à en être le parrain, le phonographe, on obtiendra des photographies de la voix...»

Le Blanc, 1877 , p. 1623

«Il paraît que cette fois le journal américain [New York Tribune] n'avait rien exagéré ; l'invention est réelle, l'instrument prodigieux existe, il s'appelle le phonographe».

Le Figaro, 14 mars 1878, p. 2

«Tout le public savant connaît aujourd'hui l'admirable appareil imaginé par M. Edison, le phonographe parlant, qui permet de reproduire la voix, le chant, et d'enregistrer et de conserver indéfiniment la parole.»

Le Moniteur universel, 6 mai 1878, p. 729

La note du $T L F$, qui situe la création du mot et du concept en 1864 sous la plume du photographe célèbre Nadar, indique très justement 1877 comme datation de la réalisation.

phonographie

1) enregistrement sonore

«Il paraît que Guillaume Weber a eu aussi, à son tour, l'idée de la phonographie; mais nous ne sommes pas en mesure de faire une citation exacte»,

Niaudet, 1878 , p. 149

«On se rappellera que j'étais seul sur le terrain de 1854 à 1859 . On jugera peut-être que cette seconde partie de ma brochure constitue une bonne leçon, neuve, je vous l'assure, de ... (comment dois-je dire, messieurs les savants?) phonographie ou de phonautographie?»

Scott, 1878 , p. 4 
2) support d'enregistrement

«...ces photographies (de la voix) qui devront prendre le nom de phonographies...»

Le Blanc, 1877 , p. 1623

voir aussi sous phonographier

Phonographie dans le premier sens est bien attesté dans le $T L F$ en 1869 , mais il est peut-être possible de reculer cette datation en dépouillant les ouvrages antérieurs de Scott de Martinville. Dans le second sens il ne semble figurer nulle part.

phonographier

"On ira se faire phonographier comme on va depuis longtemps se faire photographier, et l'on enverra à ses amis sa phonographie, au lieu de lui envoyer sa photographie.»

«La phonogravure et le gramophone», La Nature 786,1888, p. $50-51$ de 1931.

Le $T L F$ qualifie phonographier de «vx et rare», mais on peut reculer la datation

\section{phonographique}

«... vous pouvez avoir dans votre salon un phonographe avec un casier contenant un album de matières phonographiques triées sur le volet.»

Le Figaro 12 avril 1878 , p. 1

Voir aussi cliché, phonogramme.

La première attestation est une traduction de l'anglais, ce qui conforte l'intuition du $T L F$, qui suggère un emprunt à l'anglais, notant phonographic en anglais (1878, contre 1935 en français), mais les autres attestations suggèrent une dérivation directe de phonographe.

\section{reproduction}

«L'Académie a entendu sortir du fond du cornet la reproduction de toutes les paroles sorties de la bouche de l'opérateur $[\ldots]$ »,

L'Illustration, 1830, 3 mars 1878, p. 196

reproduction mécanique

«L'inscription et la reproduction mécaniques de la voix humaine» titre.

La Nature, 786, 1888, p. 49

reproduire

«Le phonographe d'Edisson [sic] servant à reproduire la parole» (titre)

L'Illustration, 1830, 3 mars 1878, p. 196

«Devant la Société de physique de Londres avec un phonographe à deux embouchures, on a pu clicher et ensuite reproduire un duo.»

L'Illustration, 1831, 30 mars 1878, p. 210

sillon

«Il se compose [...] d'un simple cylindre horizontal en cuivre, sur lequel on a pratiqué un sillon hélicoïdal. Toutes les parties du sillon viennent successivement se dérouler devant une pointe traçante implantée sur un ressort placé au fond d'un porte-voix.»

L'Illustration, $1830,3.03 .1878$, p. 196

«MM Bell et Tainter /.../ emploient un stylet tranchant qui grave ou découpe sur un cylindre, recouvert de cire d'abeille ou de paraffine, un sillon à peine visible.»

J. Wetzler, La lumière électrique, 11.10.1888, p. 290

Sans datation dans le $T L F$, sillon est classé dans l'audiovisuel, la première attestation étant de 1958. 


\section{RÉFÉRENCES}

1. Ouvrages et articles de linguistique

GUILBERT, Louis (1967): Le vocabulaire de l'astronautique, Rouen, Publications de l'Université de Rouen, $361 \mathrm{p}$.

MORTUREUX, Marie-Françoise (s.d.) : «Paradigmes désignationnels», SEMEN, 8, Annales littéraires de l'Université de Besançon, pp. 121-142.

TOURNIER, Jean (1985): Introduction descriptive à la lexicogénétique de l'anglais contemporain, ParisGenève, Champion-Slatkine, $517 \mathrm{p}$.

WOOLDRIDGE, Terence (1990-1991): «Vocabulaires néologiques dans une revue d'art», Halifax, ALFA, $3 / 4$, pp. 89-116.

WÜSTER, Eugen (1985) : Einführung in die allgemeine Terminologielehre und Terminologische Lexikographie, $2^{\mathrm{e}}$ édition, Infoterm / Handelshojskolen i Kobenhavn, $214 \mathrm{p}$.

1.1 Dictionnaire

Trésor de la langue française (TLF), Dictionnaire de la langue du XIX et du XX $X^{e}$ siècle (1978-1992): sous la direction de P. Imbs, puis de B. Quemada, Paris, Éd. du CNRS, Klincksieck, Gallimard, 15 volumes parus.

\section{Sources primaires}

2.1 Articles anonymes (par ordre chronologique)

article, Le Figaro, 14 mars 1878, p. 2.

article, Le Figaro, 12 avril 1878, p. 1.

«Le phonographe d'Edison au boulevard des Capucines», La Nature, 267, 4 mai 1878, pp. 366-367.

article, Le Moniteur universel, 6 mai 1878, p. 729

«Le phonographe», Le Magasin pittoresque, 46, octobre 1878, pp. 343-344.

article, La lumière électrique, 32, 25 mars 1888, p. 358.

«La phonogravure et le gramophone», La Nature, 786, 1888, pp. 49-51 .

\subsection{Articles signés}

FONVIELLE, W. de, article, L'Illustration, 1830, 3 mars 1878, p. 196.

FONVIELLE, W. de, article, LIllustration 1831,30 mars 1878, p. 210

FONVIELLE, W. de, article, L' Illustration 1833, 13 avril 1878, p. 245.

HOSPITALIER, E. (1879) : «Les lames parlantes de M. Lambrigot», La Nature, 309, 3 mai 1879, pp. 349-350.

LE BLANC (= LENOIR) (1877) : «Le téléphone et le phonographe», La semaine du clergé, 51, 10 octobre 1877 , pp. $1623-1625$.

LE BLANC (= LENOIR) (1878) : «Le monde des sciences et des arts», La semaine du clergé, XI, 14, 23 janvier 1878, p. 440.

LE BLANC (= LENOIR) (1878) : «Grosse erreur à rectifier dans l'histoire du phonographe», La semaine du clergé, 20 novembre 1878 , pp. 149-151.

RICHARD, Gustave (1886) : «Les graphophones», La lumière électrique, 23 novembre 1886, pp. 299-305.

RICHARD, Gustave (1889) : «Les graphophones», La lumière électrique, 32, 25 mai 1889, pp. 358-360.

WETZLER, J. (1887) : «États-Unis», La lumière électrique, 10 décembre 1887, p. 542.

WETZLER, J. (1888) : «États-Unis», La lumière électrique, 11 octobre 1888, pp. 290-292.

\subsection{Monographies}

NIAUDET, Alfred (s.d.): Téléphones et phonographes. Étude complète de ces inventions, 24 gravures dans le texte, Paris, J. Baudry. s. d. Acquisition Bibliothèque de l'Arsenal 1878, 176 p.

Il peut paraître hasardeux d'inclure dans une étude de datations un livre qui n'est pas daté. Compte tenu des informations techniques qu'il contient, cependant, il ne peut être antérieur à 1878 , et puisqu'il a été acquis par la Bibliothèque de l'Arsenal cette année-là, il s'agit nécessairement de l'année de la publication.

SCOTT DE MARTINVILLE, E. Léon (1878): Le problème de la parole s'écrivant elle-même; La France 1853-1861-1877; L'Amérique 1877-1878, Première édition provisoire, Paris, chez l'auteur, mai 1878.

\section{Sources secondaires: histoire du phonographe}

CAIMI, Daniel, BINARI, Gianfranco [1977] (1984): Stereostor : Un secolo di riproduzione sonora, Gruppo Editoriale Suno, Rome.

CHARBON, Paul (1981): La machine parlante, Paris, Éditions Jean-Pierre Gys, 208 p

GELATT, Roland (1956) : The Fabulous Phonograph, London, Cassel and Company, Londres, $250 \mathrm{p}$. 